\title{
MOLIENDA DE ZANAHORIA: UNA ESTRATEGIA PARA PRODUCIR ALIMENTOS CON VALOR AGREGADO
}

\section{Carrot milling: a strategy for value-added food production.}

\author{
${ }^{1}$ Cristian Patiño-Vidal* (iD , ${ }^{1}$ Cristina Muñoz-Shugulí iD , ${ }^{2}$ Marcelo Patiño-Vidal ${ }^{\text {iD }},{ }^{3}$ Jorge Ocampo- \\ Jiménez (iD), ${ }^{2}$ Eduardo Muñoz-Jácome (iD \\ ${ }^{1}$ Universidad de Santiago de Chile, Facultad Tecnológica, Departamento de Ciencia y Tecnología de \\ los Alimentos, Chile. \\ ${ }^{2}$ Escuela Superior Politécnica de Chimborazo, Facultad de Mecánica/ Facultad de Recursos Naturales, \\ Escuela de Ingeniería Industrial/ Escuela de Ingeniería Agronómica, Ecuador. \\ ${ }^{3}$ La Universal, Departamento de Mejora Continua, Ecuador \\ *cristian.patino@usach.cl
}

$\mathrm{R}$ esumen

La molienda es una operación unitaria ampliamente utilizada en la industria alimentaria para transformar granos, vegetales y carnes, a productos con tamaño pequeño. En este sentido, en el presente trabajo se diseñó y construyó un molino de martillos a pequeña escala con el objetivo de reducir el tamaño de zanahoria deshidratada y así, obtener un alimento con valor agregado. Los parámetros del molino como fuerza, torque y potencia se determinaron mediante cálculos teóricos, mientras que los parámetros operacionales como coeficiente de molienda y rendimiento se determinaron mediante el proceso de molienda y tamizado de la zanahoria. La fuerza necesaria del equipo para moler la zanahoria fue $5820 \mathrm{~kg}{ }^{\star} \mathrm{m} / \mathrm{s}^{2}$, mientras que el torque y potencia requerida por el motor para generar los cortes a través de los martillos fueron $34,92 \mathrm{~kg}^{\star} \mathrm{m}^{2} / \mathrm{s}^{2} \mathrm{y}$ $989,28 \mathrm{~kg}^{\star} \mathrm{m}^{2} / \mathrm{s}^{3}$, respectivamente. Un rendimiento del $90 \%$ y un coeficiente de molienda de 10 permitió reducir el diámetro de la zanahoria desde $5 \mathrm{~cm}$ hasta $0,5 \mathrm{~cm}$. Estos resultados demuestran que el equipo diseñado y construido en este trabajo permite reducir el tamaño de zanahoria deshidratada, proceso que da valor agregado al alimento y que se podría consumir como un snack natural.

Palabras clave: Molienda, molino de martillos, snack, industria de alimentos.

\section{A bstract}

Milling is a unit operation widely used in the food industry to turn grains, vegetables and meats into small-sized products. In this context. In this sense, a small-scale hammer mill was designed and built in order to reduce the size of dehydrated carrots and thus obtain a value-added food. Mill parameters such as force, torque and power were determined by theoretical calculations, while operational parameters such as milling coefficient and performance were determined by the carrot milling and sieving process. The force required by the equipment to mill the carrot was $5820 \mathrm{~kg}{ }^{\star} \mathrm{m} / \mathrm{s} 2$, whereas the torque and power required by the motor to generate the cuts through the hammers were $34.92 \mathrm{~kg}^{\star} \mathrm{m} 2 / \mathrm{s} 2$ and $989.28 \mathrm{~kg}^{\star} \mathrm{m} 2 / \mathrm{s} 3$, respectively. A performance of $90 \%$ and a milling coefficient of 10 allowed to reduce the diameter of the carrot from $5 \mathrm{~cm}$ to $0.5 \mathrm{~cm}$. These results show that the equipment designed and built in this work allows to reduce the size of dehydrated carrot, a process that gives added value to the food and could be consumed as a natural snack.

Keywords: Milling; hammer mill; snack; food industry. 


\section{INTRODUCCIÓN}

La molienda es una operación unitaria ampliamente utilizada en la industria minera, farmacéutica, agricultura, papel y de alimentos, la cual permite reducir el tamaño de piezas grandes a partículas pequeñas con un tamaño definido, mediante la aplicación de golpes, presiones de cizallamiento o corte (1-3). En el área de los alimentos, esta técnica se ha utilizado desde la antigüedad por los primeros homínidos que utilizaban herramientas de piedra para cortar principalmente granos, raíces y madera, hasta la actualidad mediante el uso de máquinas industriales que permiten obtener productos más elaborados como harinas, balanceados, alimentos instantáneos, entre otros (4-6). Así, diferentes equipos como quebrantadores, trituradoras, máquinas de corte y molinos, como el de martillos, son los más utilizados en la industria para reducir el tamaño de varios alimentos (7).

El molino de martillos es un equipo que contiene un disco rotor compuesto por una serie de pequeñas piezas de corte denominados "martillos" que giran a alta velocidad en el interior de una carcasa cilíndrica, con la finalidad de romper o trocear la alimentación o carga que ingresa por la parte superior de dicha carcasa $(8,9)$. El diseño de este tipo de máquinas está determinado tanto por las características del alimento a procesar tales como tamaño inicial y final, forma, friabilidad o capacidad de ruptura, así como por las características de operación requeridas por el equipo como fuerza, torque, potencia, grado de desintegración, entre otros $(10,11)$. El uso del molino de martillos en la industria de alimentos tiene una gran presencia. Esto debido a que su aplicación está involucrado en empresas multinacionales de productos altamente comercializados como las cervecerías, fábricas de harinas o piensos (12). En estas empresas, la preferencia del molino de martillos frente a otro tipo de máquinas como el molino de piedra, molino de discos, molino de cuchillas o molino de chorros se debe principalmente a las ventajas que ofrece tales como: i) la obtención de partículas con tamaños muy homogéneos (13), ii) la capacidad de trabajar con altas velocidades de alimentación (12), iii) la capacidad de molienda continua con grandes cantidades de alimento y altos rendimientos del producto (14), y iv) el resguardo de las propiedades activas del alimento (15). De acuerdo con esto, diversas investigaciones han centrado sus objetivos en el diseño y construcción de este tipo de molinos con potencial aplicación en alimentos como el maíz o los cereales. Por ejemplo, Atarama (2018) realizó la sistematización del diseño de un molino de martillos con la finalidad de producir harina a partir de granos de maíz (16). Asimismo, Verdesoto (2015) reportó el diseño de un molino de martillos con la capacidad de procesar $250 \mathrm{~kg} / \mathrm{h}$ de maíz para producir balanceados en granjas avícolas (17). De igual manera, Chávez y Ramos (2018) diseñaron un molino de martillos con la finalidad de procesar $500 \mathrm{~kg} / \mathrm{h}$ de cereales y forraje (18).

Se puede evidenciar que los trabajos mencionados solamente se enfocaron en la molienda de granos para la producción de harinas y su posterior consumo. Por esa razón, el diseño y construcción de un molino de martillos y su posterior aplicación para la obtención de productos como los snacks, es una estrategia que permitiría agregar valor a diferentes alimentos.

Los snacks generalmente son alimentos que tienen un gran valor comercial por su facilidad de manipulación y consumo, pero un reducido valor nutricional debido a su alto procesamiento $(19,20)$. Sin embargo, en los últimos años la mayoría de investigaciones se han enfocado en la obtención de alimentos más nutritivos, fáciles de manipular $y$, sobre todo, mínimamente procesados $(21,22)$. De acuerdo con un análisis realizado por ProChile, en el 2017 se estimó que el mercado mundial de los snacks se encontraba por encima de los 375.000 millones de dólares, de los cuales el $50 \%$ de este valor correspondía a la venta de snacks naturales y un $30 \%$ a la venta de snacks orgánicos (23). Por lo tanto, como los gustos y preferencias del consumidor se ha direccionado a alimentos más naturales en los últimos años, la zanahoria se constituiría en un alimento de gran interés que podría ser usado para el desarrollo de snacks naturales debido a su bajo costo y alto valor nutritivo (24). La zanahoria (Daucus carota $L$.) es uno de los alimentos más cultivados y consumidos a nivel mundial. Según la platafor- 
ma FAOSTAT de la Organización de las Naciones Unidas para la Alimentación y la Agricultura, en 2016 se estimó una producción de 42,7 millones de toneladas de zanahoria en el mundo (25), y en varios estudios se describe que esta enorme demanda se debe a su valor nutritivo (26).

Por otro lado, para el desarrollo de un snack a partir de zanahoria, es necesario primero deshidratar el alimento y posteriormente reducir su tamaño, con el fin de llevarlo a un formato versátil para el consumidor. Por ese motivo, el uso de un molino de martillos constituye una excelente alternativa, siendo necesario su diseño y construcción para la producción a pequeña escala. Además, como el uso de este tipo de maquinaria podría inicialmente beneficiar a pequeños y medianos productores de zanahoria en Ecuador, la presente investigación se inscribió dentro del proyecto "Definición de alternativas productivas ecosociales para el manejo sustentable de la microcuenca del río Chimborazo", ya que en la parte baja de esta microcuenca, en las parroquias San Andrés, San Juan y Santiago de Calpi, el cultivo de zanahoria ocupa una superficie mayor a 100 hectáreas (27-29). De esta forma, la producción de snacks naturales permitiría dar valor agregado a este importante producto primario de la provincia de Chimborazo, y por otra parte, se generaría emprendimientos sostenibles con la capacidad de ser replicados en otras latitudes de la sierra ecuatoriana, donde el cultivo de la zanahoria ocupa superficies mucho mayores, incidiendo positivamente también en tres de las prioridades más importantes de Ecuador tales como: el cambio de la matriz productiva, el comercio justo y la generación de empleo a través del fomento del emprendimiento (30).

En base a estos antecedentes se ejecutó la presente investigación, con el objetivo de diseñar y construir un molino de martillos a pequeña escala que permita agregar valor al cultivo de zanahoria cuando se reduce su tamaño.

\section{MATERIALES Y MÉTODOS}

\section{A. Materiales}

Como materia prima se utilizó zanahoria Dau- cus carota L. de la variedad Chantenay Red Core, obtenida de los mercados populares "Mayorista", "La Condamine", "San Alfonso" y "Dávalos" de la ciudad de Riobamba en la provincia de Chimborazo en Ecuador. Por otro lado, se utilizó acero inoxidable AISI $304 \mathrm{~L} 2 \mathrm{~B}$ como material de construcción del molino de martillos.

\section{B. Obtención y preparación del material vegetal}

Para la preparación de las muestras se siguió el protocolo establecido por Beltrán y Veloz (2015) (31). Las zanahorias se cortaron en rodajas de 2 $\mathrm{cm}$ de espesor y $5 \mathrm{~cm}$ de diámetro, se secaron a $65{ }^{\circ} \mathrm{C}$ durante 1 hora en un secador de bandejas tipo armario y se pesaron periódicamente en una balanza digital Radwag (WLC-6/A2, Polonia) hasta obtener un peso constante. La muestra deshidratada (200 gramos) se almacenó en un ambiente libre de humedad hasta su uso.

\section{Datos teóricos del molino de martillos}

Los cálculos de ingeniería requeridos para el dimensionamiento del molino de martillos se realizaron tomando en cuenta las siguientes consideraciones:

- Una capacidad de molienda de zanahoria seca de $10 \mathrm{~kg} / \mathrm{h}$, requerimiento de un equipo de baja escala (32).

- Un diámetro final de partícula de $5 \mathrm{~cm}$, con el fin de facilitar la manipulación y consumo del producto tipo snack por parte del consumidor. - Una densidad del alimento de $1110 \mathrm{~kg} / \mathrm{m} 3$, calculada mediante el método de desplazamiento de volumen.

De acuerdo con esta información, se establecieron los datos teóricos para los martillos, tolva de alimentación y motor del molino, los cuales se muestran en la Tabla 1 y en las Figuras 1 y 2

\begin{tabular}{lcc}
\hline \multicolumn{1}{c}{ Especificación técnica } & Unidad & Valor \\
\hline Distancia entre los martillos frontales & $\mathrm{m}$ & 0,006 \\
Número de martillos & - & 16,00 \\
Velocidad angular del motor & $\begin{array}{c}\text { Revoluciones } \\
\text { por segundo }\end{array}$ & 28,33 \\
Ancho de la tolva de alimentación & $\mathrm{m}$ & 0,150 \\
Longitud de la tolva de alimentación & $\mathrm{m}$ & 0,250 \\
Altura de la tolva de alimentación & $\mathrm{m}$ & 0,030 \\
\hline
\end{tabular}

Tabla 1. Datos teóricos para el dimensionamiento del molino de martillos. 
A)

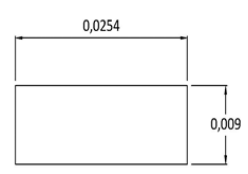

C)

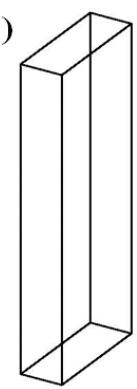

Figura 1. Vistas de diseño: A) superior, B) frontal, y C) 3D de un martillo del molino.
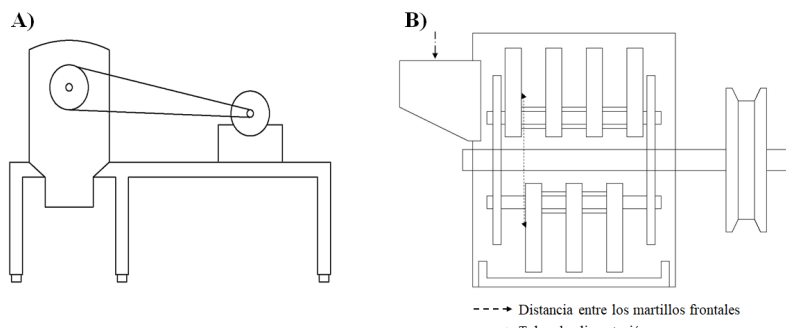

Figura 2. Vistas de diseño: A) lateral, y B) interna del molino de martillos.

D. Cálculo de los parámetros teóricos y operacionales del equipo

1) Parámetros teóricos

El área de entrada a cada martillo $\left(A_{e}\right)$ y el volumen de la tolva de alimentación $\left(\mathrm{V}_{\mathrm{t}}\right)$ para la zona de alimentación se determinaron mediante las ecuaciones 1 y 2 , respectivamente.

$$
\begin{gathered}
A_{e}=a^{\star} l \\
V_{t}=A^{\star} L^{\star} H
\end{gathered}
$$

Donde: $a y l$ es el ancho y largo de cada martillo, mientras que $A, L y H$ es el ancho, largo y la altura de la tolva de alimentación.

La fuerza requerida $(\mathrm{F})$ del molino de martillos basado en un esfuerzo de corte de un material vegetal deshidratado $\sigma_{m v}=3 \times 106 \mathrm{~kg} / \mathrm{m}^{*} \mathrm{~s}^{2}$ se determinó mediante la ecuación 3 (33).

$$
F=A_{e}^{*} \sigma_{m v}
$$

El torque $(\mathrm{T})$ y potencia $(\mathrm{W})$ requerido por el motor del molino se calcularon mediante las ecuaciones 4 y 5 , respectivamente.

$$
\begin{aligned}
T & =F^{\star} r \\
W & =T^{*} \omega
\end{aligned}
$$

Donde: $r$ es la distancia entre los martillos frontales y $\omega$ es la velocidad angular del motor.

\section{2) Parámetros operacionales}

Una vez diseñado el equipo mediante los cálculos teóricos mencionados, se construyó un mo- lino de martillos a pequeña escala. La zanahoria deshidratada se molió durante 5 minutos en el equipo, y se tamizó en tamices con luz de malla de $1,0,75$ y $0,5 \mathrm{~cm}$. Además, el producto neto $\left(P_{n}\right)$ y porcentaje de rechazo $(\% R)$ se determinaron mediante las ecuaciones 6 y 7 , respectivamente.

$$
\begin{aligned}
& P_{n}=P_{i}-P_{\text {finos }} \\
& \% R=P_{f} / P_{n}
\end{aligned}
$$

Donde: $P_{i}$ es el peso inicial de la zanahoria con un diámetro de $5 \mathrm{~cm}, P_{\text {finos }}$ es el peso de la zanahoria molida con un tamaño menor a $0,5 \mathrm{~cm}, y$ $P_{f}$ es el peso final de la zanahoria molida con un tamaño igual a $0,5 \mathrm{~cm}$.

Finalmente, el rendimiento y el coeficiente de molienda $(\Sigma)$ del molino de martillos se determinaron mediante las ecuaciones 8 y 9 , respectivamente.

$$
\begin{gathered}
R=P_{n} / P_{i} \\
\Sigma=D_{i} / D_{f}
\end{gathered}
$$

Donde: $D_{i}$ es el diámetro inicial y $D_{f}$ el diámetro final de la zanahoria molida.

\section{RESULTADOS Y DISCUSIÓN}

A. Parámetros teóricos del molino de martillos La Tabla 2 muestra los parámetros teóricos calculados para el dimensionamiento del molino de martillos. Se obtuvo una carga máxima de zanahoria deshidratada de $0,0011 \mathrm{~m}^{3}\left(\mathrm{~V}_{\mathrm{t}}\right)$, y la fuerza requerida por la máquina para romper el material vegetal seco fue de $5820 \mathrm{~kg}{ }^{\star} \mathrm{m} / \mathrm{s}^{2}(\mathrm{~N})$. A pesar de que en la literatura existente no se evidencian datos para la molienda de zanahoria, los datos obtenidos se acercan a valores de fuerza requeridos para otros alimentos. Por ejemplo, Chávez y Ramos (2018) demostraron que una fuerza de $2087,2 \mathrm{~N}$ fue necesaria para romper cereal y forraje, mientras que Atarama (2018) calculó una fuerza de $6633,79 \mathrm{~N}$ para romper granos de maíz $(16,18)$, en ambos casos con un molino de martillos. Es evidente que la fuerza que requiere un molino para reducir el tamaño de un material está estrechamente relacionada con el esfuerzo de corte y las características propias de la muestra, como la dureza. Rastogi et al. (2008) reportaron una dureza de aproximadamente $190 \mathrm{~N}$ 
para zanahoria fresca (34). De acuerdo con ello, se observa que la zanahoria aumentó significativamente su dureza luego de deshidratarse, ya que se requirió una elevada fuerza para romperla. Este hecho está probablemente relacionado al pretratamiento de secado de la zanahoria, en donde las moléculas de agua que actúan como plastificante son desplazadas, lo cual genera un vegetal más duro (35). Sin embargo, el secado de la zanahoria con temperaturas entre 60 y $70{ }^{\circ} \mathrm{C}$ también produce un colapso en la estructura fibrilar, promoviendo un cambio en la viscosidad local (interna) de la matriz (36), lo cual puede generar un material vegetal menos fuerte que el maíz (16).

Por otra parte, el torque y la potencia necesaria del motor que permitiría girar los 16 martillos para moler la zanahoria deshidratada fueron de $34,92 \mathrm{~kg}^{\star} \mathrm{m}^{2} / \mathrm{s}^{2}$ y $989,28 \mathrm{~kg}^{\star} \mathrm{m}^{2} / \mathrm{s}^{3}$, respectivamente. El torque es la fuerza necesaria que requiere el motor del molino para hacer girar sus componentes internos (16). En efecto, el torque requerido por el motor de nuestro equipo es 2 veces menor respecto al de un molino de 32 martillos diseñado para producir harina de sorgo (37). Así también, Atarama (2018) observó que un incremento de 24 a 36 martillos en un molino para procesar maíz produjo un aumento del torque de 100 a $160 \mathrm{~kg}^{*} \mathrm{~m}^{2} / \mathrm{s}^{2}$ (16). Por otra parte, altas cargas de alimentación en el equipo conllevan al uso de motores más potentes, $\mathrm{y}$ viceversa. Al comparar la capacidad de molienda del molino de martillos para producir harina de sorgo $(173 \mathrm{~kg} / \mathrm{h})(37)$ respecto a la capacidad de nuestro molino $(10 \mathrm{~kg} / \mathrm{h})$, se observa que la potencia requerida para moler la zanahoria deshidratada es 17 veces menor. En definitiva, tanto el torque como la potencia requerida de un motor en un molino de martillos se relacionan directamente con el número de martillos y la capacidad de carga, respectivamente.

\begin{tabular}{lcc}
\multicolumn{1}{c}{ Parámetro } & Unidad & Valor \\
\hline Área de entrada a cada martillo & $\mathrm{m}^{2}$ & 0,0019 \\
Volumen de tolva de alimentación & $\mathrm{m}^{3}$ & 0,0011 \\
Fuerza & $\mathrm{kg}^{\star} \mathrm{m} / \mathrm{s}^{2}$ & 5820,0 \\
Torque & $\mathrm{kg}^{*} \mathrm{~m}^{2} / \mathrm{s}^{2}$ & 34,920 \\
Potencia & $\mathrm{kg}^{*} \mathrm{~m}^{2} / \mathrm{s}^{3}$ & 989,28
\end{tabular}

Tabla 2. Parámetros teóricos calculados para el molino de martillos

\section{B. Parámetros operacionales del molino de martillos}

Luego del proceso de molienda y tamizado, la cantidad de producto con un tamaño mayor o igual al deseado se define como el producto neto molido $\left(P_{n}\right)$, la relación entre la cantidad de producto con el tamaño deseado $\left(P_{f}\right)$ y la cantidad de producto neto molido se define como el porcentaje de rechazo $(\% R)$, y la cantidad de producto con un tamaño menor al deseado se define como producto fino $\left(P_{\text {finos }}\right)$ (38). En la presente investigación, se diseñó y construyó un molino de martillos con la capacidad de reducir el tamaño de zanahoria deshidratada a $0,5 \mathrm{~cm}$. La Tabla 3 muestra los resultados de los parámetros operacionales del equipo luego de procesar la zanahoria. Posterior a los procesos de molienda y tamizado de los 200 gramos de zanahoria, el valor de $P_{f} y P_{\text {finos }}$ fue 0,164 y $0,021 \mathrm{~g}$, respectivamente. De acuerdo con estos resultados, la cantidad de $P_{n}$ obtenido fue de $0,179 \mathrm{~g}$ con un porcentaje de rechazo del 91,62\%, operando el molino de martillos con un rendimiento del $90 \%$. Estos resultados evidenciaron que los parámetros de diseño utilizados anteriormente permitieron construir un molino de martillos altamente eficiente para reducir el diámetro de la zanahoria deshidratada desde $5 \mathrm{~cm}$ a $0,5 \mathrm{~cm}$.

Por otro lado, la relación entre los tamaños de entrada y salida de las muestras durante el proceso de molienda se denomina como coeficiente de molienda $(\Sigma)$, cuyo valor puede estar entre 2 y 15 (39). Valores cercanos a 2 indican que el equipo no está cumpliendo con las especificaciones de diseño, mientras que valores cercanos a 15 afirman su correcto funcionamiento. La Tabla 3 muestra que se obtuvo un valor de $\Sigma$ igual a 10 luego del proceso de molienda de la zanahoria deshidratada, demostrando que el molino de martillos cumplió con las especificaciones iniciales establecidas en su diseño.

\begin{tabular}{lcc}
\hline \multicolumn{1}{c}{ Parámetro } & Unidad & Valor \\
\hline Producto neto & $\mathrm{kg}$ & 0,179 \\
Porcentaje de rechazo & $\%$ & 91,62 \\
Rendimiento & $\%$ & 90,00 \\
Coeficiente de molienda & - & 10,00 \\
\hline
\end{tabular}

Tabla 3. Parámetros operacionales del molino de martillos 


\section{CONCLUSIONES}

Se diseñó y construyó un molino de martillos a pequeña escala con la capacidad de reducir el tamaño de zanahoria deshidratada desde $5 \mathrm{~cm}$ hasta $0,5 \mathrm{~cm}$. Un menor número de martillos $\mathrm{y}$ una menor capacidad de molienda produjo que los valores del torque y potencia requeridos por el motor del equipo sean menores respecto a los valores que se requieren en un motor de un molino para producir harina de sorgo. El alto valor de porcentaje de rechazo evidenció que el 91,62\% del producto neto molido mantuvo un diámetro de $0,5 \mathrm{~cm}$, mientras que un rendimiento del 90 $\%$ demostró que el molino fue capaz de procesar los 200 gramos de zanahoria deshidratada casi en su totalidad.

\section{AGRADECIMIENTOS}

Los autores agradecen al Ing. Hannibal Brito Moina y al Laboratorio de Operaciones Unitarias de la Facultad de Ciencias de la Escuela Superior Politécnica de Chimborazo.

R eferencias

1. Barderas AV, Obaya Valdivia AE. Operaciones Unitarias para la Industria Alimentaria (Un enfoque basado en competencias) [Internet]. Cuautitlán; 2016 [cited 2020 Apr 14]. 535 p. Available from: https://s3.amazonaws.com/academia.edu.documents/56515559/OperacionesUnitariasparalaIndustriaAlimentaria.pdf?response-content-disposition=inline\%3B filename\%3DUNIVERSIDAD_NACIONAL_AUTONOMA_DE_MEXICO.pdf\&X-Amz-Algorithm=AWS4-HMAC-SHA256\&X-Amz-Credential=ASI

2. Kotamarthy L, Metta N, Ramachandran R. Understanding the effect of granulation and milling process parameters on the quality attributes of milled granules. Processes [Internet]. 2020 Jun 1 [cited 2020 Aug 27];8(6):683. Available from: www.mdpi.com/journal/processes

3. Seibert KD, Collins PC, Luciani C V., Fisher ES. MILLING OPERATIONS IN THE PHARMACEUTICAL INDUSTRY. In: Chemical Engineering in the Pharmaceutical Industry [Internet]. Hoboken, NJ, USA: John Wiley \& Sons, Inc.; 2019 [cited 2020 Aug 27]. p. 861-79. Available from: http://doi. wiley.com/10.1002/9781119600800.ch38

4. Pawar T, Pavan Kumar P, Ashwin Kumar MS, Jyothi Lakshmi A, Sakhare SD, Dasappa I, et al. Milling interventions for the production of atta for Indian flat bread with low carbohydrate digestibility. J Food Sci Technol [Internet]. 2020 Sep 11 [cited 2020 Aug 27];57(9):3400-8. Available from: http:// link.springer.com/10.1007/s13197-020-04373-x

5. Cappelli A, Oliva N, Cini E. Stone milling versus roller milling: A systematic review of the effects on wheat flour quality, dough rheology, and bread characteristics [Internet]. Vol. 97, Trends in Food Science and Technology. Elsevier Ltd; 2020 [cited 2020 Aug 27]. p. 147-55. Available from: https:// linkinghub.elsevier.com/retrieve/pii/S092422441930980X

6. Gao W, Chen F, Wang X, Meng Q. Recent advances in processing food powders by using superfine grinding techniques: A review. Compr Rev Food Sci Food Saf [Internet]. 2020 Jul 1 [cited 2020 Aug 27];19(4):2222-55. Available from: https://onlinelibrary.wiley.com/doi/full/10.1111/1541-4337.12580

7. Salas Lara CG. Diseño de un molino de rodillos para molienda de granos, destinado al área de alimentos de CESTTA-ESPOCH [Internet]. [Riobamba]: Escuela Superior Politécnica de Chimborazo; 2012 [cited 2020 Apr 14]. Available from: http://dspace.espoch.edu.ec/bitstream/123456789/1982/1/96T00161.pdf

8. Ojomo OA, Fawohunre AJ. Development of a Hammer Mill with Double Sieving Screens. Eur J Eng Res Sci [Internet]. 2020 May 31 [cited 2020 Aug 27];5(5):617-21. Available from: https://www.ejers. org/index.php/ejers/article/view/1763

9. Sumardi S, Nurjaman F, Isnugroho K, Junaedi A, Handoko AS. The alloy steel from manganese-chrome-copper as an alternative material to make hammer mill hammers. In: PROCEEDINGS OF THE 3RD INTERNATIONAL SEMINAR ON METALLURGY AND MATERIALS (ISMM2019): Exploring New Innovation in Metallurgy and Materials [Internet]. AIP Publishing; 2020 [cited 2020 Aug 27]. p. 060009. Available from: http://aip.scitation.org/doi/abs/10.1063/5.0001925 
10. Lyu F, Thomas M, Hendriks WH, van der Poel AFB. Size reduction in feed technology and methods for determining, expressing and predicting particle size: A review. Vol. 261, Animal Feed Science and Technology. Elsevier B.V.; 2020. p. 114347.

11. Cortazar-Figueroa LM, Meléndez-Pérez R, Oliver-Hernández DM. Consumo de energía y distribución de tamaño de partícula en la molienda de canela (Cinnamomum zeylanicum) y pimienta negra (Piper nigrum L). Rev Mex Ing química [Internet]. 2008 [cited 2020 Apr 14];7(2):123-30. Available from: http://www.scielo.org.mx/scielo.php?script=sci_arttext\&pid=S1665-27382008000200004

12. Ezurike BO, Osazuwa OJ, Okoronkwo CA, Okoji KI. Design, construction and performance evaluation of a flat screen hammer mill machine. African J Sci Technol Innov Dev [Internet]. 2018 Sep 19 [cited 2021 May 6];10(6):683-90. Available from: https://www.tandfonline.com/doi/abs/10.1080/204 21338.2018.1491675

13. Selle PH, Truong HH, Khoddami A, Moss AF, Roberts TH, Liu SY. The impacts of hammer-mi11 screen size and grain particle size on the performance of broiler chickens offered diets based on two red sorghum varieties. Br Poult Sci [Internet]. 2019 Dec 9 [cited 2021 May 6];60(3):209-18. Available from: https://www.tandfonline.com/action/journalInformation?journalCode=cbps20

14. Lee D, Kim MJ, Kwak HS, Kim SS. Characteristics of bread made of various substitution ratios of bran pulverized by hammer mill or jet mill. Foods [Internet]. 2020 Jan 4 [cited 2021 May 6];9(1):48. Available from: www.mdpi.com/journal/foods

15. Polari JJ, Garcí-Aguirre D, Olmo-García L, Carrasco-Pancorbo A, Wang SC. Impact of industrial hammer mill rotor speed on extraction efficiency and quality of extra virgin olive oil. Food Chem [Internet]. 2018 Mar 1 [cited 2021 May 6];242:362-8. Available from: https://pubmed.ncbi.nlm. nih.gov/29037701/

16. Atarama Valdiviezo LA. Sistematización de diseño de molinos de martillos fijos para grano [Internet]. [Piura]: Universidad de Piura; 2018 [cited 2020 Apr 14]. Available from: https://alicia.concytec.gob.pe/vufind/Record/UDEP_6ff40fbb7817d0607edb693f42d5681d

17. Verdesoto García MF. Diseño de un molino artesanal para maíz con una capacidad de $250 \mathrm{~kg} / \mathrm{h}$ para granjas avícolas [Internet]. [Quevedo]: Universidad Técnica de Quevedo; 2015 [cited 2020 Apr 1]. Available from: http://repositorio.uteq.edu.ec/bitstream/43000/1415/1/T-UTEQ-0010.pdf

18. Chávez Vela FH, Ramos Vásquez G. Diseño de un molino de martillos para cereales y forraje de $500 \mathrm{~kg} / \mathrm{h}$ en el anexo de Batangrande, Lambayeque [Internet]. [Pimentel]: Universidad Señor de Sipán; 2018 [cited 2020 Apr 1]. Available from: http://200.60.28.26/bitstream/handle/uss/4949/Chávez Vela \%26 Ramos Vasquez.pdf?sequence $=1$ \&isAllowed $=y$

19. Hepworth AD, Moding KJ, Stifter CA. A laboratory-based assessment of mother-child snack food selections and child snack food consumption: Associations with observed and maternal self-report of child feeding practices. Food Qual Prefer [Internet]. 2020 Jul 1 [cited 2020 Aug 27];83:103898. Available from: https://linkinghub.elsevier.com/retrieve/pii/S0950329319306123

20. Setiyowati E, Alamsyah S. Effect Of Health Education About Snack Foods On Snack Food Preferences In Elementary School Students At SDN Jagir 1/393 Surabaya. J Ners dan Kebidanan Indones [Internet]. 2020 Apr 30 [cited 2020 Aug 27];7(3):136. Available from: http://ejournal.almaata.ac.id/ index.php/JNKI

21. Soleno Wilches R. Tecnologías no térmicas en el procesado y conservación de alimentos vegetales. Rev Colomb Investig agroindustriales. 2015 Dec 24;2:73.

22. Sant'Anna PB, Melo Franco BD, Maffei DF. Microbiological safety of ready-to-eat minimally processed vegetables in Brazil: an overview. J Sci Food Agric [Internet]. 2020 Oct 8 [cited 2020 Nov 12];100(13):4664-70. Available from: https://onlinelibrary.wiley.com/doi/abs/10.1002/jsfa.10438

23. ProChile. Tendencias de Snacks Saludables en Ecuador [Internet]. 2017 Nov [cited 2021 May 13]. Available from: https://www.prochile.gob.cl/wp-content/uploads/2017/11/ficha_tendencias_snacks_saludables_ecuador_2017.pdf

24. Gebhardt SE, Thomas RG. Nutritive Value of Foods [Internet]. 2002 [cited 2020 Apr 14]. Available from: https://naldc.nal.usda.gov/download/CAT11131126/PDF

25. Keser D, Guclu G, Kelebek H, Keskin M, Soysal Y, Sekerli YE, et al. Characterization of aroma and phenolic composition of carrot (Daucus carota 'Nantes') powders obtained from intermittent mi- 
crowave drying using GC-MS and LC-MS/MS. Food Bioprod Process. 2020 Jan 1;119:350-9.

26. Janiszewska-Turak E, Witrowa-Rajchert D. The influence of carrot pretreatment, type of carrier and disc speed on the physical and chemical properties of spray-dried carrot juice microcapsules. Dry Technol [Internet]. 2020 Feb 8 [cited 2020 Apr 14];1-11. Available from: https://www.tandfonline. com/doi/full/10.1080/07373937.2019.1705850

27. Cholota A. Plan de Desarrollo y Ordenamiento Territorial de la Parroquia San Andrés [Internet]. San Andrés; 2014 [cited 2020 Apr 20]. Available from: https://www.gadsanandres.gob.ec/images/ PDOT SAN ANDRES 2015.pdf

28. Fernández Vinueza DF. Actualización Plan de Desarrollo y Ordenamiento Territorial Parroquia San Juan [Internet]. San Juan; 2015 [cited 2020 Apr 20]. Available from: http://app.sni.gob.ec/snilink/sni/PORTAL_SNI/data_sigad_plus/sigadplusdocumentofinal/0660823500001_ACTUALIZACIÒN PDYOT SAN JUAN 2015-2019_30-10-2015_22-28-53.pdf

29. Paca Alguasaca JA. Actualización del Plan de Desarrollo y Ordenamiento Territorial Parroquia de Santiago de Calpi [Internet]. Santiago de Calpi; 2015 [cited 2020 Apr 20]. Available from: http://app. sni.gob.ec/sni-link/sni/PORTAL_SNI/data_sigad_plus/sigadplusdocumentofinal/0660821800001_ Calpi ultimo_30-10-2015_21-08-14.pdf

30. Moreno L. Plan Nacional de Desarrollo 2017-2021-Toda una Vida [Internet]. Quito; 2017 [cited 2020 Apr 20]. Available from: https://www.planificacion.gob.ec/wp-content/uploads/downloads/2017/10/PNBV-26-OCT-FINAL_0K.compressed1.pdf

31. Beltrán Cabrera TP, Veloz Gavilanez SA. Diseño y Construcción de un Secador tipo Armario para la deshidratación hasta el diez por ciento de Berro, Espinaca, Zanahoria [Internet]. [Riobamba]: Escuela Superior Politécnica de Chimborazo; 2015 [cited 2020 Mar 31]. Available from: http://dspace. espoch.edu.ec/handle/123456789/3654

32. Calderón De Zacatares V, López LM, Duville KE, Bermúdez CE. Utilizacion de Molinos CTI para la Produccion Artesanal de Harina de Sorgo. INTSORMIL Sci Publ [Internet]. 2012 [cited 2021 May 13]; Available from: http://digitalcommons.unl.edu/intsormilpubs

33. Zambrano Santana LR. Diseño de una maquina picadora de rastrojo, para producción de abono organico [Internet]. [Guayaquil]: Escuela Superior Politécnica del Litoral; 2004 [cited 2020 Mar 31]. Available from: https://www.dspace.espol.edu.ec/handle/123456789/14653

34. Rastogi NK, Nguyen LT, Balasubramaniam VM. Effect of pretreatments on carrot texture after thermal and pressure-assisted thermal processing. J Food Eng. 2008 Oct;88(4):541-7.

35. Rajkumar G, Shanmugam S, Galvâo M de S, Leite Neta MTS, Dutra Sandes RD, Mujumdar AS, et al. Comparative evaluation of physical properties and aroma profile of carrot slices subjected to hot air and freeze drying. Dry Technol [Internet]. 2017 Apr 26 [cited 2021 May 13];35(6):699-708. Available from: https://www.tandfonline.com/doi/abs/10.1080/07373937.2016.1206925

36. Xu C, Liu D, Li Y, Li G, Zhang J, Gao R. Implication of water state on glass transition temperature in hot air-dried carrot slices. LWT - Food Sci Technol. 2017 Oct 1;84:780-7.

37. Monteagudo Castillo YR. Diseño de un molino de martillo para la obtención de harina de sorgo [Internet]. [Santa Clara]: Universidad Central Marta Abreus de las Villas; 2016 [cited 2020 Apr 1]. Available from: http://dspace.uclv.edu.cu/bitstream/handle/123456789/7177/Monteagudo Castillo Yandry Reinaldo.pdf?sequence $=1$ \&isAllowed $=y$

38. Samaniego Toapanta MX, Estrada Yambay EW. Diseño y construcción de un equipo mixto de molienda y tamizado para materiales minerales [Internet]. [Riobamba]: Escuela Superior Politécnica de Chimborazo; 2012 [cited 2020 Apr 8]. Available from: http://dspace.espoch.edu.ec/bitstream/123456789/1973/1/96T00152.pdf

39. Patiño Vidal CJ, Ocampo Jiménez JA. Diseño y construcción de un equipo reductor de tamaño de berro, espinaca, zanahoria [Internet]. [Riobamba]: Escuela Superior Politécnica de Chimborazo; 2014 [cited 2020 Apr 8]. Available from: http://dspace.espoch.edu.ec/bitstream/123456789/3877/1/96T00282 UDCTFC.pdf 